\title{
A Case of Pneumatic Displacement with Gas Tamponade Performed for Macular Subretinal Hemorrhage Complicating Vogt-Koyanagi-Harada Disease
}

\author{
Takatoshi Kobayashi $^{a} \quad$ Masanori Fukumoto ${ }^{a}$ Nanae Takai ${ }^{b}$ \\ Kohichi Maruyama $^{c}$ Rei Tada ${ }^{d}$ Hiromi Shouda $^{a}$ Teruyo Kida ${ }^{a}$ \\ Tsunehiko Ikeda ${ }^{a}$ \\ ${ }^{\text {aD }}$ epartment of Ophthalmology, Osaka Medical College, Takatsuki-City, Japan; ${ }^{\text {bTakai Eye }}$ \\ Clinic, Kawachinagano-City, Japan; 'Maruyama Eye Clinic, Takatsuki-City, Japan; ${ }^{\mathrm{d}}$ Tada Eye \\ Clinic, Ikeda-City, Japan
}

\section{Keywords}

Subretinal hemorrhage $\cdot$ Choroidal neovascularization · Vogt-Koyanagi-Harada disease . Intravitreal gas injection P Pneumatic displacement

\begin{abstract}
We experienced a case of subretinal hemorrhage (SRH) from choroidal neovascularization (CNV) complicating Vogt-Koyanagi-Harada disease (VKH) that underwent pneumatic displacement of hematoma by intravitreal gas injection. A 76-year-old male revealed VKH relapses and optical coherence tomography showed irregular retinal pigment epithelium in his right eye and serous retinal detachment and retinal pigment epithelial detachment in his left eye. Fluorescein angiography of the left eye showed hyperfluorescence possibly attributable to CNV. One month later, SRH occurred in the left eye, yet it was spontaneously absorbed. However, approximately 1 year later, the SRH recurred in the left eye affecting a wide area, including the macular region, and his visual acuity (VA) decreased to 0.06 . When pneumatic displacement of
\end{abstract}


the hematoma by intravitreal gas injection was performed, the SRH was inferiorly displaced, and his VA improved to 0.4. Pneumatic displacement with gas tamponade was effective for treating a case of SRH caused by persistent CNV complicating VKH.

(c) 2020 The Author(s)

Published by S. Karger AG, Basel

\section{Introduction}

Vogt-Koyanagi-Harada disease (VKH) is associated with various complications, including cataract and glaucoma [1]. Reported macular complications include macular edema and choroidal neovascularization (CNV), and patients with persistent VKH are known to be at an increased risk of developing these macular complications [2-4]. Although the intravitreal injection of anti-vascular endothelial growth factor (VEGF) agents is reportedly useful for treating patients with VKH complicated by CNV [5, 6], CNV may be exacerbated in patients in whom treatment with anti-VEGF agents is contraindicated for various reasons.

Here we report a case of VKH complicated by CNV. In this case, even though the patient was followed up with only local steroid therapy, instead of anti-VEGF therapy, because he had systemic vaso-occlusive diseases, he suffered subretinal hemorrhage from CNV and was subsequently treated with pneumatic displacement with gas tamponade.

\section{Case Report}

This study involved a 76-year-old male patient who had previously visited a local hospital 2 years earlier when both eyes already showed sunset-glow fundus. At that time, serous retinal detachment (SRD) was noted, and steroid pulse therapy was administered based on the diagnosis of VKH relapse. Prior to that visit, the patient had not noticed any particular visual impairment suggestive of the onset of $\mathrm{VKH}$. Although the condition of the fundi then remained stable, VKH once again relapsed 1 year later, SRD and retinal pigment epithelial detachment (RPED) occurred, and his corrected visual acuity (VA) decreased to 0.09. For treatment, steroid pulse therapy was once again administered at that local hospital, and his corrected VA recovered to 0.7. However, 3 months later, VKH relapsed for the third time, and his corrected VA decreased to 0.3 . Thus, the patient was subsequently referred to our department. His past medical history included diabetes mellitus, coronary stenting for myocardial infarction, and iliac artery bypass using prosthetic graft for iliac artery occlusion.

\section{Findings at the Initial Visit to Our Department}

SRD and RPED were observed in the patient's left eye. In his right and left eye, the VA was $0.5\left(0.8 \times \mathrm{S}-0.5 \mathrm{D}=\mathrm{C}-1.0 \mathrm{D} \mathrm{Ax} 100^{\circ}\right)$ and $0.45\left(0.5 \times \mathrm{C}-0.5 \mathrm{D}\right.$ Ax $\left.110^{\circ}\right)$, respectively, and the intraocular pressure was 13 and $15 \mathrm{~mm} \mathrm{Hg}$, respectively. Slit-lamp microscopy revealed an intraocular lens implanted in the right eye and a moderate nuclear cataract in the left eye. Both eyes showed a sunset-glow fundus (Fig. 1a, b), and optical coherence tomography (OCT) showed irregular retinal pigment epithelium in the macular region of the right eye and SRD and RPED in the macular region of the left eye (Fig. 2a, b). Fluorescein angiography showed hyperfluorescence due to window defect in an area from the optic disc to the macular region in the left eye and hyperfluorescence presumably due to CNV (Fig. 3). Indocyanine green angiography also showed hyperfluorescence presumably due to CNV at the same site (Fig. 4). Based on the above findings, CNV secondary to persistent VKH was diagnosed. 
Clinical Course of Treatment

The patient was followed up with ophthalmic administration of steroids and subtenon injection of triamcinolone acetonide. Since he had systemic vaso-occlusive diseases, he did not consent to anti-VEGF therapy and it was subsequently not administered. At 6 weeks after his initial visit, macular subretinal hemorrhage occurred in the left eye (Fig. 5a), and his left-eye VA decreased to 0.3. OCT showed RPED and SRD in the macular region of the left eye (Fig. 5b). Subsequently, the subretinal hemorrhage was spontaneously absorbed, and the corrected VA improved to 0.6. However, 3 years after his initial visit, macular subretinal hemorrhage recurred in the left eye (Fig. 6a), and his left-eye corrected VA decreased to 0.06. OCT showed RPED and hemorrhagic RD in the macular region of the left eye (Fig. 6b). Since the volume of the hemorrhage was large, the patient was admitted to our department, received an injection of $0.4 \mathrm{~mL}$ of $100 \%$ sulfur hexafluoride $\left(\mathrm{SF}_{6}\right)$ gas into the vitreous cavity, and was instructed to lie in the prone position. Two days after the gas injection, the macular subretinal hemorrhage was inferiorly displaced, and then was spontaneously, yet gradually, absorbed. His left-eye corrected VA then improved to 0.4 (Fig. 7), and OCT findings showed relief of the SRD and RPED (Fig. 8).

\section{Discussion and Conclusion}

Patients with persistent VKH are known to be at an increased risk of developing CNV [24]. Read et al. [3] reported that CNV did not occur in patients with VKH treated with adequate systemic steroid therapy from the early stages, but did appear in $11 \%$ of patients with persistent VKH. Yang et al. [4] reported that the incidence of macular complications (i.e., macular edema and CNV) was $5.7 \%$ in patients who received adequate initial treatment with steroids and immunosuppressants for VKH, but as high as $13.1 \%$ in patients who did not receive adequate treatment. In our present case, since the patient already had sunset-glow fundi at his initial visit to the previous local hospital, VKH might have occurred earlier and resolved spontaneously without being much noticed by the patient. Thus, no initial treatment was administered, and he appears to have been at a risk of developing persistent VKH.

Inomata et al. [7] reported the detailed pathogenic mechanism of CNV complicating VKH. In that study, they histopathologically examined CNV that complicated VKH 26 years after the onset and reported that $\mathrm{CNV}$ associated with the proliferation of pigment epithelium was observed in the peripheral fundus. In chronic uveitis, CNV may occur in the peripheral fundus, in the macular region, and around the optic disc. As for the cause, damage to the Bruch's membrane and choriocapillaris induces ischemia, which stimulates vascular endothelial and pigment epithelial cells of the choriocapillaris and leads to CNV.

Although anti-VEGF therapy is the treatment that is usually selected for CNV, there are reports of treatment with bevacizumab and other anti-VEGF agents administered for CNV complicating VKH $[5,6]$. However, and as described above, since infiltration of inflammatory cells into the choroid is a possible cause of $\mathrm{CNV}$, many reports indicate that steroid therapy should be administered in combination with anti-VEGF therapy. Ranjan et al. [8] reported a case in which bevacizumab and a steroid were injected into the vitreous body to treat CNV complicating $\mathrm{VKH}$. Although the $\mathrm{CNV}$ in that case completely regressed, rebound inflammation of VKH occurred and the patient was subsequently treated with oral administration of steroids and immunosuppressants. Pai et al. [9] also reported that a combination of steroids and anti-VEGF agents is useful for the management of CNV in repeatedly relapsing VKH. In addition, it has been reported that photodynamic therapy was effective $[10,11]$, and that CNV was 
removed by vitreous surgery [12]. In our present case, since the patient had a history of systemic vaso-occlusive diseases (i.e., myocardial infarction and iliac artery occlusion), he did not consent to the use of anti-VEGF agents. Thus, only local steroid therapy was administered for the CNV. It appears that the CNV consequently persisted and led to the subretinal hemorrhage.

Reportedly, the common types of uveitis that cause CNV are thought to include punctate inner choroidopathy, multifocal choroiditis, and VKH [13]. CNV complicating uveitis is often difficult to diagnose due to poor mydriasis, vitreous opacity, and cystoid macular edema, etc., and therefore, treatment is highly likely to be delayed [14]. For the diagnosis, indocyanine green angiography, in addition to OCT and fluorescein angiography, is useful [15]. As for fundus features, in addition to multiple choroidal scar lesions, macular scars, scars around the optic disc, and subretinal fluid, a subretinal hemorrhage may lead to the detection of the CNV. Although subretinal hemorrhage due to a rupture of the CNV seems relatively rare, Bansal et al. [14], who examined 73 eyes of 60 patients with uveitis complicated by CNV, reported that subretinal hemorrhage was detected in 5 eyes. However, and as far as we know, there have been no previously reported cases in which pneumatic displacement with gas tamponade was performed for submacular hematoma, as in our present case. Like in cases of age-related macular degeneration, when macular retinal hematoma persists, it not only damages retinal photoreceptors and neurons, but also triggers subretinal fibroplasia [16-18]. Thus, reducing the thickness of the hematoma by gas tamponade in the early stages and promoting early absorption appear to be important for maintaining good VA.

\section{Acknowledgement}

The authors wish to thank John Bush for editing the manuscript.

\section{Statement of Ethics}

The protocols of this study were approved by the Ethics Committee of Osaka Medical College, Takatsuki-City, Osaka, Japan.

In accordance with the tenets set forth in the Declaration of Helsinki, prior written informed consent was obtained from the patient.

\section{Disclosure Statement}

There are no conflicts of interest to report for all authors.

\section{Funding Sources}

Not applicable. 


\section{Author Contributions}

T.Ko., M.F., N.T., K.M., R.T., H.S. and T.Ki.: equal contribution of patient management, conception of the study, data analysis and interpretation of the manuscript drafting, and literature search. T.Ko. and T.Ki.: co-writing of the manuscript, literature search. T.I.: design of the study, co-writing of the manuscript, literature search, and manuscript editing and revision, as well as final approval. All authors read and approved the final manuscript.

\section{References}

1 O'Keefe GA, Rao NA. Vogt-Koyanagi-Harada disease. Surv Ophthalmol. 2017 Jan - Feb;62(1):1-25

2 Read RW, Rechodouni A, Butani N, Johnston R, LaBree LD, Smith RE, et al. Complications and prognostic factors in Vogt-Koyanagi-Harada disease. Am J Ophthalmol. 2001 May;131(5):599-606.

3 Read RW, Yu F, Accorinti M, Bodaghi B, Chee SP, Fardeau C, et al. Evaluation of the effect on outcomes of the route of administration of corticosteroids in acute Vogt-Koyanagi-Harada disease. Am J Ophthalmol. 2006 Jul;142(1):119-24.

4 Yang P, Ye Z, Xu J, Du L, Zhou Q Qi J, et al. Macular Abnormalities in Vogt-Koyanagi-Harada Disease. Ocul Immunol Inflamm. 2019;27(8):1195-1202.

5 Raffa L, Bawazeer A. Intravitreal bevacizumab injection in a 14-year-old Vogt-Koyanagi-Harada patient with choroidal neovascular membrane. Can J Ophthalmol. 2009 Oct;44(5):615-6.

6 Wu L, Evans T, Saravia M, Schlaen A, Couto C. Intravitreal bevacizumab for choroidal neovascularization secondary to Vogt-Koyanagi-Harada syndrome. Jpn J Ophthalmol. 2009 Jan;53(1):57-60.

7 Inomata H, Minei M, Taniguchi Y, Nishimura F. Choroidal neovascularization in long-standing case of VogtKoyanagi-Harada disease. Jpn J Ophthalmol. 1983;27(1):9-26.

8 Ranjan R, Agarwal M. Rebound inflammation after an intravitreal injection in Vogt-Koyanagi-Harada syndrome. Indian J Ophthalmol. 2018 Jun;66(6):863-5.

9 Pai SA, Hebri SP, Lootah AM. Management of recurrent inflammatory choroidal neovascular membrane secondary to Vogt-Koyanagi-Harada syndrome, using combined intravitreal injection of bevacizumab and triamcinolone acetate. Indian J Ophthalmol. 2012 Nov-Dec;60(6):551-2.

10 Nowilaty SR, Bouhaimed M; Photodynamic Therapy Study Group. Photodynamic therapy for subfoveal choroidal neovascularisation in Vogt-Koyanagi-Harada disease. Br J Ophthalmol. 2006 Aug;90(8):982-6.

11 Farah ME, Costa RA, Muccioli C, Guia TA, Belfort R Jr. Photodynamic therapy with verteporfin for subfoveal choroidal neovascularization in Vogt-Koyanagi-Harada syndrome. Am J Ophthalmol. 2002 Jul;134(1):137-9.

12 Foster RE, Knight CD, Lowder CY. Subfoveal choroidal neovascular membrane excision in Vogt-KoyanagiHarada syndrome. Retina. 2000;20(5):547-9.

13 Wu K, Zhang X, Su Y, Ji Y, Zuo C, Li M, et al. Clinical characteristics of inflammatory choroidal neovascularization in a Chinese population. Ocul Immunol Inflamm. 2016 Jun;24(3):261-7.

14 Bansal R, Bansal P, Gupta A, Gupta V, Dogra MR, Singh R, et al. Diagnostic challenges in inflammatory choroidal neovascular membranes. Ocul Immunol Inflamm. 2017 Aug;25(4):554-62.

15 Stanga PE, Lim JI, Hamilton P. Indocyanine green angiography in chorioretinal diseases: indications and interpretation: an evidence-based update. Ophthalmology. 2003 Jan;110(1):15-21.

16 Read RW, Rao NA, Cunningham ET Jr. Vogt-Koyanagi-Harada disease. Curr Opin Ophthalmol. 2000 Dec;11(6):437-42.

17 Lertsumitkul S, Whitcup SM, Nussenblatt RB, Chan CC. Subretinal fibrosis and choroidal neovascularization in Vogt-Koyanagi-Harada syndrome. Graefes Arch Clin Exp Ophthalmol. 1999 Dec;237(12):1039-45.

18 Sheu SJ, Kou HK, Chen JF. Prognostic factors for Vogt-Koyanagi-Harada disease. J Chin Med Assoc. 2003 Mar;66(3):148-54. 


\section{Case Reports in Ophthalmology}

\begin{tabular}{l|l}
\hline Case Rep Ophthalmol 2020;11:143-150 \\
\hline DOI: 10.1159/000506436 & $\begin{array}{l}\text { @ 2020 The Author(s). Published by S. Karger AG, Basel } \\
\text { www.karger.com/cop }\end{array}$ \\
\hline
\end{tabular}

Kobayashi et al.: Subretinal Hemorrhage Complicating Vogt-Koyanagi-Harada Disease

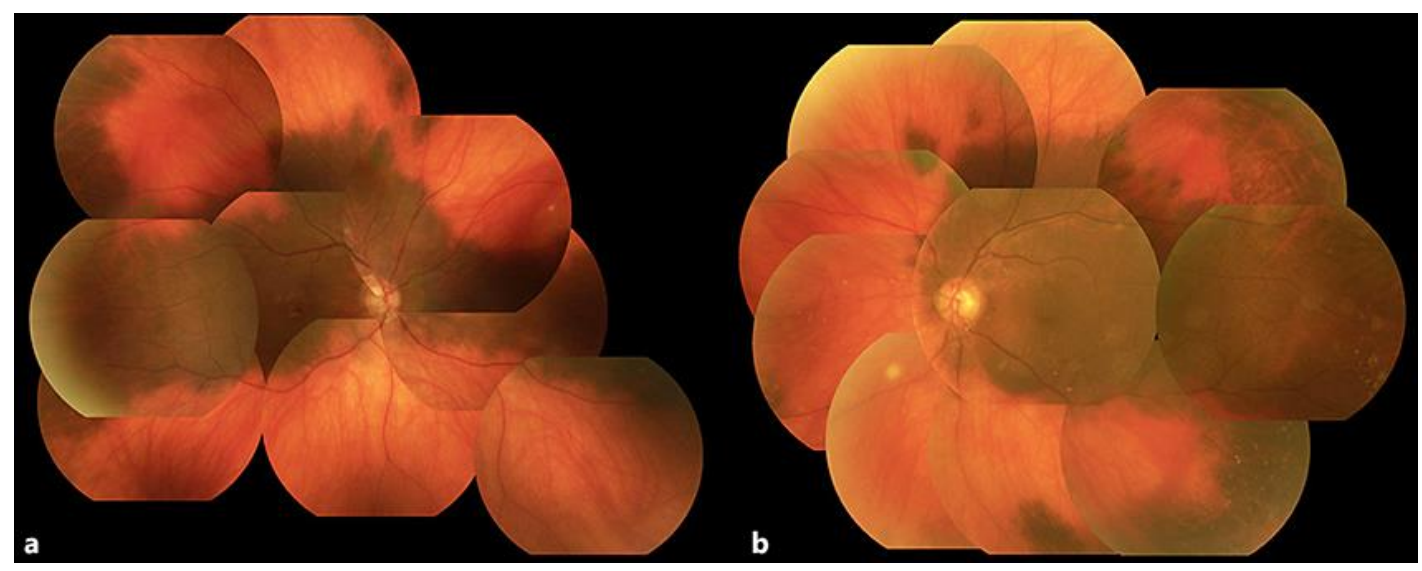

Fig. 1. Fundus photographs obtained at the initial examination (a: right eye, b: left eye) showing sunsetglow fundus in both eyes.
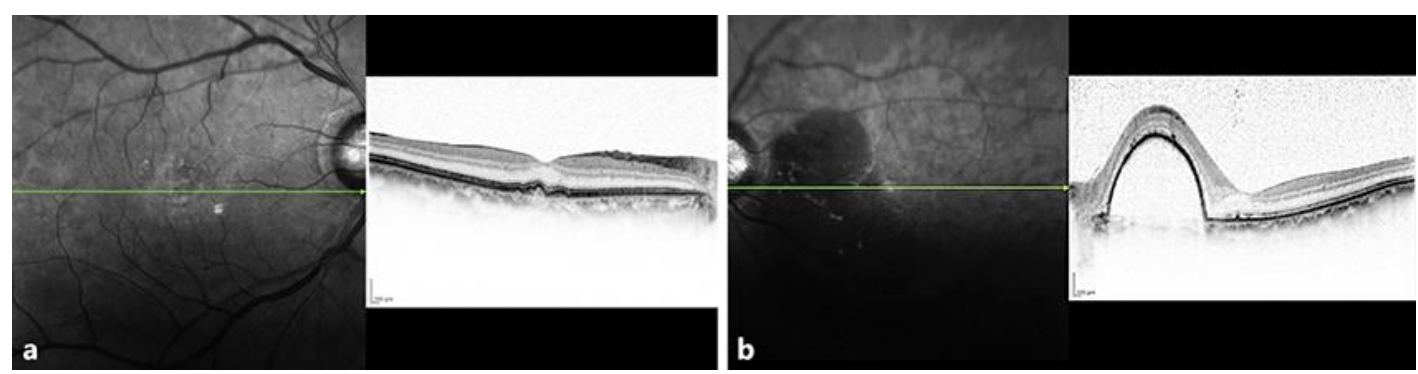

Fig. 2. OCT images obtained at the initial examination showing irregular retinal pigment epithelium in the macular region in the right eye (a), and SRD and retinal RPED in the macular region of the left eye (b).

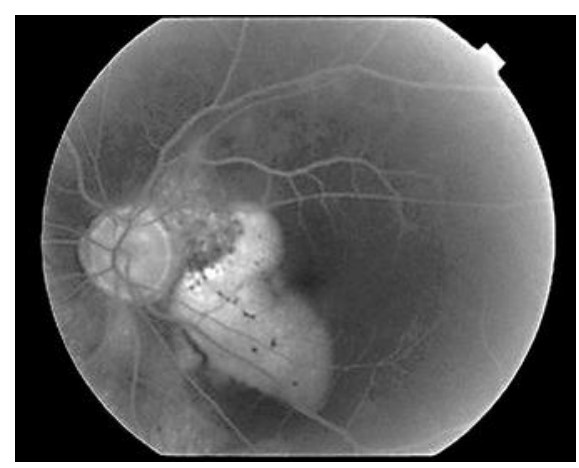

Fig. 3. Fluorescein angiography image of the patient's left eye obtained at the initial examination showing hyperfluorescence due to window defect in an area from the optic disc to the macular region and hyperfluorescence presumably due to CNV. 


\section{Case Reports in Ophthalmology}

\begin{tabular}{l|l}
\hline Case Rep Ophthalmol 2020;11:143-150 \\
\hline DOI: 10.1159/000506436 & $\begin{array}{l}\text { @ 2020 The Author(s). Published by S. Karger AG, Basel } \\
\text { www.karger.com/cop }\end{array}$ \\
\hline
\end{tabular}

Kobayashi et al:: Subretinal Hemorrhage Complicating Vogt-Koyanagi-Harada Disease

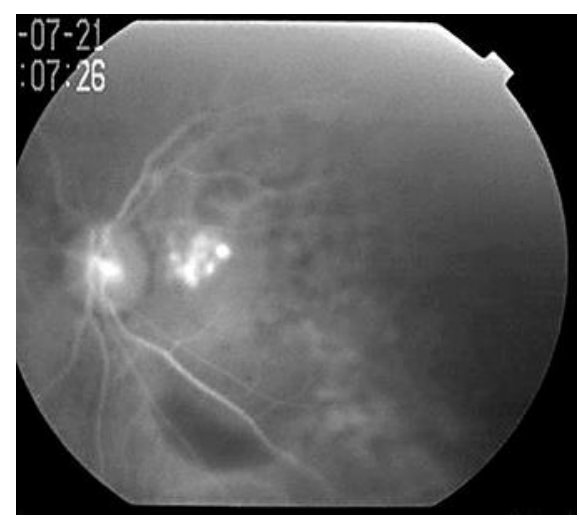

Fig. 4. Indocyanine green angiography image of the patient's left eye obtained at the initial examination showing hyperfluorescence presumably due to CNV in an area from the optic disc to the macular region.
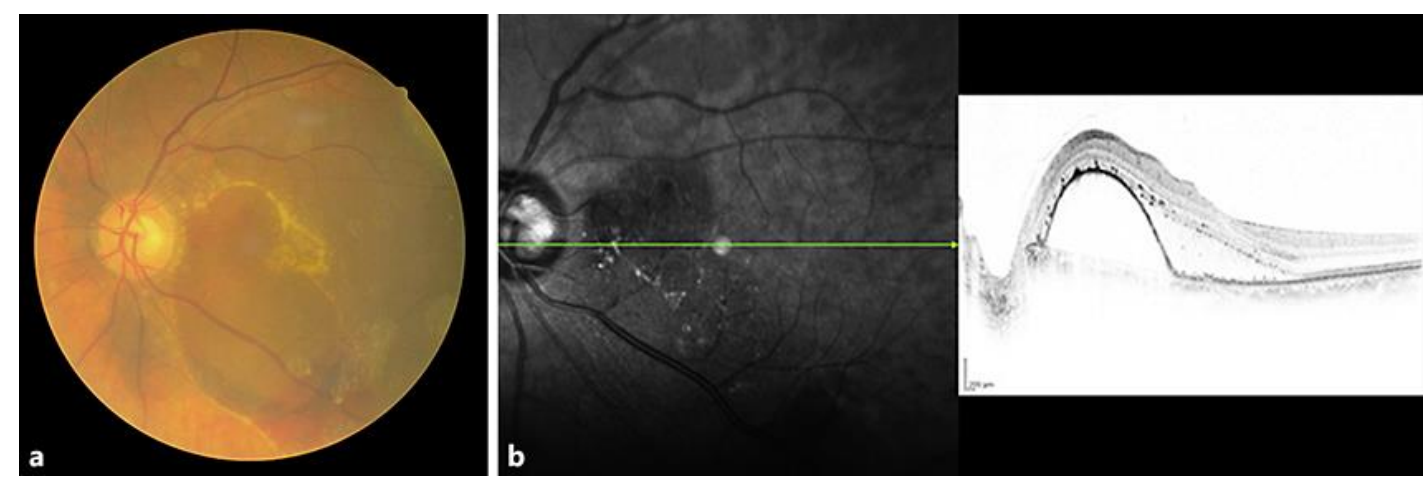

Fig. 5. Fundus photograph (a) and OCT image (b) of the patient's left eye obtained at 6 weeks after his initial presentation. Although macular subretinal hemorrhage occurred, it was spontaneously absorbed.
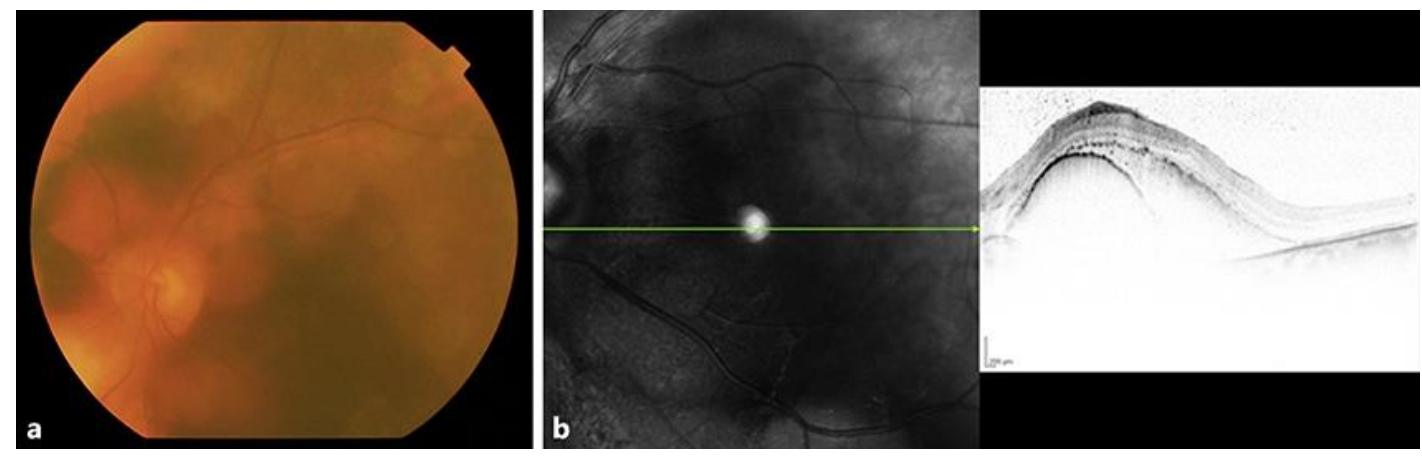

Fig. 6. Fundus photograph (a) and OCT image (b) of the patient's left eye obtained at 3 years after his initial presentation showing recurrence of the macular-region subretinal hemorrhage. 


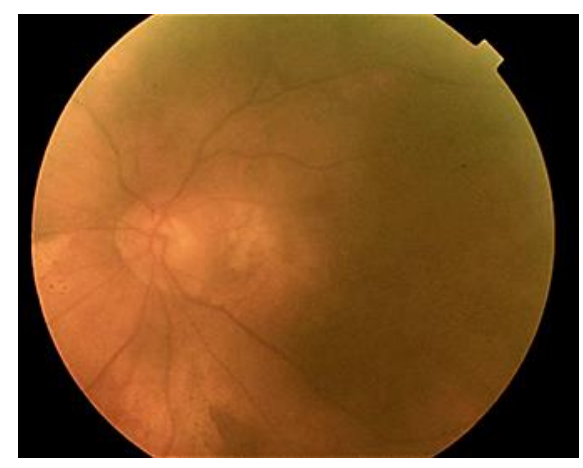

Fig. 7. Fundus photograph of the patient's left eye obtained at 2 days after the gas injection. The macularregion subretinal hemorrhage was inferiorly displaced, and was then spontaneously, yet gradually, absorbed.

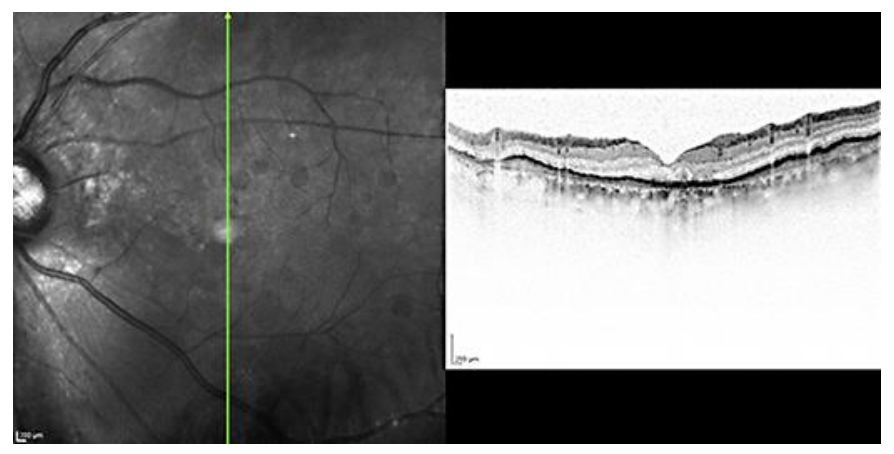

Fig. 8. OCT image of the patient's left eye obtained at 2 days after the gas injection showing relief of the SRD and RPED. 